\title{
Study and realization of centralized monitoring system of information communication
}

\author{
Bingli Pan ${ }^{1}$, Kehang Xu' ${ }^{1}$, Hong Zhang ${ }^{1}$, Chuan You ${ }^{1}$ \\ 1. SouthWest China Branch of State Grid Corporation of China, Sichuan 620010
}

Key words: working conditions of devices; information devices; centralized monitoring

\begin{abstract}
There are many devices in information communication rooms of power supply company, where related device types and manufacturers have covered many famous manufacturers in the current market. With continuous development of power information construction, device types and quantities in information rooms have been increasing. To take effective management on working condition of these devices, the paper designs and realizes centralized monitoring system of information communication for large-scale computer rooms, realizing data acquisition and centralized data management of working condition of information communication devices and power equipment and environment. Through fusion processing of acquired device working condition information to make unified distribution to each monitoring system and then reduce repetitive work, improve work efficiency to better guarantee stable operation of many monitoring systems and information systems.
\end{abstract}

\section{Research background and significance}

\subsection{Research background}

There are many devices in information communication rooms of power supply company, where related device types and manufacturers have covered many famous manufacturers in the current market. With continuous development of power information construction, device types and quantities in information rooms have been increasing. To take effective management on working condition of these devices, various information device management, devices of power equipment and environment and monitoring systems are putting into operation, in which many monitoring systems need to acquire real-time running data from these information devices ${ }^{[1]}$.

The existing data acquisition of working condition information of the information devices are independently acquired by each system and there hasn't been unified acquisition system of working condition of information devices offering data support for every monitoring system. At the same time, with continuous development of power information construction, expansion of power network business requirements and increasing information device types, the device indicators for every monitoring business system are also constantly changing. Once the acquisition devices and indicators changed, information devices would need operators and maintainers to make manual configuration with heavy workload and long response time. At the same time, every monitoring system need to adjust the same equipment and indicators respectively, which has low efficiency and high repeatability.

In order to change the situation, it's necessary to reduce repetitive work, improve work efficiency and guarantee the stable operation of information system better.

\subsection{Research Significance}

Electric Power Company undertakes the construction, repairing and maintenance work of all electric power equipment in city and electric power, as the basic link of the national economy, has great significance for production and life of enterprises and people. Many daily businesses of Electric ${ }^{[2]}$ Power Company is inseparable from the support of information network. only by normal operation of core equipment room of information communication network, can they guarantee normal operation of their information network and of daily businesses of electric 
power company ${ }^{[3]}$.

Therefore, we should establish the centralized monitoring system of information communication in power rooms, enhance monitoring and management of information devices in computer rooms and implement online monitoring system of computer rooms to improve safety and stability of device operation in computer rooms; to realize the timeliness of information collection and processing and unified processing of working condition of information devices, reduce repetitive work, improve work efficiency and guarantee the stable operation of information system better.

\section{Research Methods and Content}

\subsection{Research Content}

The centralized monitoring system of information communication acquires related data of power equipment and environment and data in operation of information devices (internet devices, server and storage device) of large operation room of Power Supply Company, establishes sharing database of working condition and completes subscription and distribution of working condition data, and then provide working condition information for other application systems. ${ }^{[4]}$

(1)Data acquisition.Acquisition program collects status data from information devices and data of power equipment and environment, which include working status information such as data of power equipment and environment, data of server device, data of network equipment and security data.

$\checkmark$ Data of power equipment and environment: including detailed information of air conditioner, UPS detailed information and temperature and humidity information in computer rooms.

$\checkmark$ Device data of server: name of server, description of server, operating system type, CPU utilization, memory utilization, name of network card, IP address of network interface, network interface broadband, port inflow speed and port outflow speed.

$\checkmark$ Network device data: including device name, device description, IP address, operating system type, operating system description, manufacturer, CPU utilization, memory utilization, port inflow speed, port outflow speed, interface description, interface management status, interface operation status, interface broadband, interface physical address, interface IP address and interface mask, etc.

$\checkmark$ Security data: illegal outreach alarm number, system weak password information count, complement rate, desktop terminal registration rate, terminal antivirus software installation rate and implementation rate of sensitive information check.

(2)Data Storage. Constructing sharing database structure of working condition data and building infrastructure for sharing data of working condition data.

(3)Subscription and distribution of working condition data. The system can realize the subscription and distribution of working condition data, which requires functions of message receiving and sending, message subscription, information construction, message generation, message query, message filtering and monitoring of working condition data.

(4)Data reception and presentation. The application platform connects distribution system through status receiving system and the receiving pushes status data via status distribution system. Status receiving system would receive messages positively pushed by status pushing system after connecting status distribution system. The status receiving system displays the information on visual platform after receives message.

\subsection{Logical Structure of the System}

The centralized monitoring system framework of information communication uses mature and reliable JAVA technology including JMS, XML, CORBA, JDBC, RMI and ORMM apping as bases and divide modules and levels in line with functions of the system, which 
meets requirements of the interface business of working condition acquisition of information devices as well as enable the software function to be easily realized, maintained and expanded.

The system uses J2EE-based three- (multi-) layer architecture to construct the target system and uses B/S three-layer structure to build system based on different features of businesses. The system uses Struts2+Spring3+Mybatis to complete basic structure of the system; all JMS message mechanism uses ActiveMQ5.9 technology; all third-party interface uses Restful-style WebService $^{[5]}$ technology (WINK) to provide rich and easy calling API for client manufacturers and provide real-time and accurate detailed data of all devices in production environment to guarantee the accuracy and consistency of all data.

The centralized monitoring system of information communication is divided into following subsystems from perspectives of business and function.

(1)The status acquisition subsystem and the status fusion subsystem. The status acquisition system initiates the data acquisition instruction and collects acquired data respectively to the host equipment, network equipment, the dynamic loop system and the third party and then collect them into status acquisition database. Status fusion system. The status fusion system integrates the data by extracting, sorting and calculating the data, and then store the data into the status fusion database by submitter.

(2)Status subscription and distribution subsystem. The subscription and distribution service management tool is a client end that configures the publisher and subscriber under the same LAN. In the B/S mode, communication between client and server end uses HTTP protocol to configure the server through web pages. Users can use the creation and management of subscription messages and synchronization process to monitor by management tool of subscription and distribution server, and then push messages subscribed by publishers to subscribers.

(3)Data reception and presentation. The application platform connects distribution system through status receiving system and the receiving pushes status data via status distribution system. Status receiving system would receive messages positively pushed by status pushing system after connecting status distribution system. The status receiving system displays the information on visual platform after receives message.

\subsection{Workflow of the System}

(1)The status acquisition subsystem is responsible for obtaining and archiving status information.

(2)The status information NoSQL database is used to archive status information and keep monitoring devices and monitoring indicator information.

(3)RedisKey/Value is used to store the latest status information of the indicator for quick inquiry in status distribution. The status information needs to include acquisition time due to requirement of judging the latest status.

(4)The job scheduling relationship database keeps status subscription information of the third-party manufacturers and related scheduled jobs as well as equipment of the third-party manufacturer or subscription information of the indicator.

(5)The status acquisition subsystem API provide external interface service, which means to take following management function through Restful methods.

$\checkmark$ monitoring device management

$\checkmark$ monitoring index management

$\checkmark$ plug-in management

$\checkmark$ inquiring historic state information

$\checkmark$ inquiring subscription status

(6)Message-driven method: driving status to subscribe by status acquisition, i.e. the definition of equipment and indicators is determined by status acquisition party while acquisition party can be only be subscribed.

(7)If the third-party machine crashes, adopting ActiveMessageStore mechanism to ensure 
not to loss Message.

(8)Both determination and distribution should be grasped with particle size of device.

\subsection{Brief Introduction of System Features}

\subsubsection{Status Acquisition Subsystem and Status Fusion Subsystem}

CRM-AUTO acquires data via self-defined SOCKET protocol, using interacting main client/ server model between two processes, i.e. providing corresponding service to acquire data after clients send service requests to the server and the server receives the requests.

Acquiring detailed operating data of exclusive network line (DDN, VPN), network device, host device and storage device through various application layer protocols of SNMP, WMI, SYSLOG and IPMI (including ICMP, HTTP, FTP, TELNET, SMTP and POP3).

The status fusion system integrates the data by extracting, sorting and calculating the data, and then store the data into the status fusion database by submeter.

The acquisition system is responsible for acquiring and archiving status information. After processing, using ORACLE database to archive status information. Meanwhile, saving monitoring device and monitoring index information.

RedisKey/Value with very high cache efficiency is used to store the latest status information of the indicator for quick inquiry in status distribution.

Message-driven method: driving status to subscribe by status acquisition, i.e. the definition of equipment and indicators is determined by status acquisition party while acquisition party can be only be subscribed. If the third-party machine crashes, adopting MSMQ persistence framework to ensure not to loss Message. Both data storage and distribution should be with particle size of device.

\subsubsection{Status Subscription and distribution subsystem}

The user group against publishing subscription system is the third party (short for client). Because every client may subscribe different messages, the module provides management functions of clients, including subscriber list, subscriber addition, subscriber modification, subscriber delete, inquiry list of subscription device indicator, subscription device indicator, modification of subscription device indicator and inquiring historic situation of subscription device indicator. Realizing addition, delete and modification of client and making related safety certification in access to client and prevent illegal third party from accessing.

The message sending module can filter conditions of messages based on the actual situation. Due to different data resources acquired by publishing subscription system, it's necessary to determine the priority of message sending based on requirements and sending time-sensitive message in priority.

Data subscription and distribution technology offers exchange and integration of data. Data subscription and distribution service is a information sharing mechanism to realize information dynamic exchange among different resources under internet environment, including sending and accepting subscription requirements, automatically acquiring changing data, distributing contents subscribed by users, automatically maintaining environment and data within the system and providing distributed data subscription and distribution service for the whole network.

Users can publish data to several targeted servers from a data source through data subscription and distribution technology to enable users on different server to share the data within permitted scope. The data subscription and distribution technology enables automatic synchronization of databases distributed in different locations to ensure data consistency.

\subsubsection{Data Receiving Display Subsystem}

Status data uses positive pushing method by status distribution system, where status distribution system positively sends data to the subscriber and the data is transmitted in JSON format. The status distribution system acquires data in acquisition database and then pushes it 
on application platform via special network.

Application platform receives status data pushed by status distribution system. After status receiving system connects status distribution system, it would receive information positively pushed by status distribution system. The application platform save them into database by classification after processing data.

Based on different classification of data, application platform will display data on visual platform.

\subsection{Critical Technology of the System}

(1)High integration of working condition data. The real-time working condition data of acquisition information device should be quickly acquired and centralized. Automatic quality control measures should ensure correctness and effectiveness of data and process real-time working condition data according to certain procedures and standard.

(2)The unified distribution interface of working condition data. Through research and development standard interface, providing functions of real-time operating data acquisition of information device, device indicator subscription and description for the third party that needs working condition data of information devices and provide unified data acquisition and distribution interface service for system monitoring.

(3)The working condition data is convenient to customize and safe to distribute. Users or other applications can customize their necessary types, issuing time and format of receiving message via client. Distribution of working condition data based on message middleware is stable and reliable.

(4)Service-oriented Architecture (SOA). As a method of architecture and organize IT base structure and business function, Service-oriented Architecture (SOA) is an architecture design rule for resolve business application in combination with IT resources. Based on component design and service-oriented core thought, it supports turn the business into a group of interlinked service or repeatable business tasks, which is accessible via internet when necessary.

The system applies SOA to structure design of system and proposes a SOA-based workflow monitoring model in combination with workflow technology. The model combines double advantages of service-oriented architecture and workflow technology, which not only realizes function requirements of process integration, but also takes full advantages of existing resources, realizes repeated use of software, unifies interface specification and improves maintainability and scalability.

\section{Research Conclusion and Main Achievements}

\subsection{Research Conclusion}

Centralized monitoring system of information communication built by power supply company uses opening system structure based on JAVA and MQ, builds sharing database of working condition data of information devices, designs real-time database of working condition acquisition, builds sharing infrastructure of working condition data and develops interface system software for working condition acquisition of information device. The method of subscriber subscribing based on needs and the system positively pushing reduces workload of device manager and safety loophole as well as the load of monitored devices.

\subsection{Main results}

The centralized monitoring system framework of information communication uses mature and reliable JAVA technology including JMS, XML, CORBA, JDBC, RMI and ORMM aping as bases and divide modules and levels in line with functions of the system, which meets requirements of the interface business of working condition acquisition of information devices as well as enable the software function to be easily realized, maintained and expanded. 
Centralized monitoring system of information communication ensures... through acquisition, processing, subscription, broadcast and pushing system of unified status of working condition information of information device.

The application system of third-party manufacturer can acquire the status of monitored devices from the system while not directly acquire status from the monitored devices to realize repeated use of status information and reduce workload of device manager, safety loopholes as well as load of monitored devices.

The operation of centralized monitoring system of information communication in information communication center of power supply company realizes acquisition, storage, subscription and distribution of information devices and status information of dynamic loop devices, improves device operation and maintenance level of information communication center of power supply company and more fully guarantees operation of electric core business.

\section{Reference}

[1]GB/T21028 - 2007 Security technical requirements of information security technology server $[\mathrm{S}]$.

[2]HJ2507-2011 Internet server of technical requirements of environmental labeling products[S].

[3]IEC60950-23-2005IEC60950-23 , Ed.1.0 : Part 23, Information technical device. Security: Large data storage equipment [S].

[4] Joshi G, Maruvala K P. Automated software testing architecture using a multi-level framework: US, US 7664989 B2[P]. 2010.

[5] Buschmann F, Meunier R, Rohnert H, et al. Pattern-Oriented Software Architecture-A System Of Patterns[C] John Wiley \& Sons, Inc. 1996:41. 